\title{
Uncertainty Relations for Quantum Coherence
}

\author{
Uttam Singh ${ }^{1, *}$, Arun Kumar Pati ${ }^{1}$ and Manabendra Nath Bera ${ }^{2}$ \\ 1 Harish-Chandra Research Institute, Chhatnag Road, Jhunsi, Allahabad 211 019, India; akpati@hri.res.in \\ 2 ICFO-Institut de Ciències Fotòniques, The Barcelona Institute of Science and Technology, \\ ES-08860 Castelldefels, Spain; manabendra.bera@icfo.es \\ * Correspondence: uttamsingh@hri.res.in; Tel.: +91-532-2274-407; Fax: +91-532-2567-748
}

Academic Editors: Paul Busch, Takayuki Miyadera and Teiko Heinosaari Received: 4 April 2016; Accepted: 9 July 2016; Published: 16 July 2016

\begin{abstract}
Coherence of a quantum state intrinsically depends on the choice of the reference basis. A natural question to ask is the following: if we use two or more incompatible reference bases, can there be some trade-off relation between the coherence measures in different reference bases? We show that the quantum coherence of a state as quantified by the relative entropy of coherence in two or more noncommuting reference bases respects uncertainty like relations for a given state of single and bipartite quantum systems. In the case of bipartite systems, we find that the presence of entanglement may tighten the above relation. Further, we find an upper bound on the sum of the relative entropies of coherence of bipartite quantum states in two noncommuting reference bases. Moreover, we provide an upper bound on the absolute value of the difference of the relative entropies of coherence calculated with respect to two incompatible bases.
\end{abstract}

Keywords: uncertainty relations; coherence; quantum correlations

\section{Introduction}

The linearity of quantum mechanics gives rise to the concept of superposition of quantum states of a quantum system and is one of the characteristic properties that makes a clear distinction between the ways a classical and a quantum system can behave. Recently, there has been a considerable effort devoted towards quantifying quantum superposition (quantum coherence) from a resource theoretic perspective [1-31]. Also, quantum coherence has been deemed to play a key role in the emerging fields such as quantum thermodynamics [32-42] and quantum biology [43-49]. This makes the advancement of a quantitative framework for coherence even more desirable. In particular, a resource theory of coherence, developed in Ref. [2], provides a set of conditions on a real valued function of quantum states for it to be a bona fide quantifier of quantum coherence. This resource theory is based on the set of incoherent operations as the free operations and the set of incoherent states as the set of free states. The set of incoherent states and the set of incoherent operations depend crucially on the choice of basis - the reference basis-and it is determined by the experimental situation at hand. Therefore, the quantification of quantum coherence intrinsically depends on the basis that we choose to define the incoherent states and incoherent operations. This seems very unsettling as any physical quantity should not depend on some arbitrary choice of basis. However, the choice of basis is provided naturally by the experimental situation at hand.

Uncertainty relations play an important role in foundations of quantum mechanics as well as in quantum information science. After the discovery of the Heisenberg uncertainty principle [50,51], Robertson and Schrödinger proved the uncertainty relations for two incompatible observables [52,53]. Recently, two stronger uncertainty relations are proved which go beyond the Robertson-Schrödinger uncertainty relation [54]. Undoubtedly, uncertainty relations continue to play pivotal role in quantum theory as well as quantum information science [55]. In addition to the variance based uncertainty 
relation, there are entropic uncertainty relations [56-60] which capture the notion of uncertainty for two incompatible observables. Entropic uncertainty relations prove to be of fundamental and practical importance in quantum information.

In this work, we explore how the coherence of a given quantum state in one reference basis is restricted by the coherence of the same quantum state in other reference bases for single and bipartite quantum systems. For single quantum systems, the trade-offs between the coherences with respect to the mutually unbiased bases (MUBs) have been obtained in Ref. [16]. Moreover, the exact complementarity relation for the $l_{1}$ norm of coherence [2] of qubit systems with respect to three MUBs is also obtained in Ref. [16]. Here, we go beyond the restriction of the reference bases being MUBs to any set of incompatible bases. Also, we focus on the trade-off relations for bipartite quantum systems and the role of entanglement in these relations. In particular, we provide lower and upper bounds on the sum of the relative entropies of coherence of a given state with respect to two or more incompatible bases. The lower bounds on the sum of the relative entropies of coherence for single and bipartite quantum systems are facilitated through the use of entropic uncertainty relations with and without memory effects. Thus, entropic uncertainty relations may be viewed as restrictions on coherence of a quantum system in two incompatible bases. For bipartite quantum systems, we find an upper bound on the sum of the relative entropies of coherence via Lewenstein-Sanpera decomposition [61] of bipartite quantum states. We then show that this upper bound can be tightened. We also provide a non trivial upper bound on the absolute value of the differences between the relative entropy of coherence obtained in two different bases.

Let us formally introduce the measures of coherence in the framework of resource theory, that is based on the set of incoherent operations and incoherent states. First we fix a reference basis and choose it to be the computational basis $\{|i\rangle\}$ (without loss of any generality). A maximally coherent state in this basis is given by $\left|\psi_{d}\right\rangle=\frac{1}{\sqrt{d}} \sum_{i=0}^{d-1}|i\rangle$ as any other state can be created from $\left|\psi_{d}\right\rangle$ using only the set of incoherent operations. Also, the coherence of this state naturally provide a reference to gauge the coherence of other quantum states. The set of all the states of the form $\rho_{I}=\sum_{i} d_{i}|i\rangle\langle i|$, where $d_{i} \geq 0$ and $\sum_{i} d_{i}=1$, forms the set $\mathcal{I}$ of all incoherent states. Any quantum state that does not belong to the set $\mathcal{I}$ will be called a coherent state and will act as a resource. In quantum theory, a physical operation is represented by a completely positive trace preserving (CPTP) map. Any CPTP map can be expressed in terms of a set of Kraus operators $\left\{M_{k}\right\}$ such that the state $\rho$ of the system is transformed to $\rho \rightarrow \sum_{k} p_{k} \rho_{k}$, where $\rho_{k}=M_{k} \rho M_{k}^{+} / p_{k}$ and $p_{k}=\operatorname{Tr}\left[M_{k} \rho M_{k}^{+}\right]$. An operator is said to be an incoherent operator if it maps diagonal states to some other diagonal states in the reference basis. Any quantum channel whose Kraus elements are the incoherent operators for a reference basis is said to be an incoherent channel (ICPTP map) and such an ICPTP map $\Lambda_{I}$ satisfies $\Lambda_{I}[\mathcal{I}] \subseteq \mathcal{I}$. In the following, we list the conditions for a real valued function of quantum states that make the function a bona fide measure of quantum coherence. Following Ref. [2], a function on the quantum state $C^{(i)}(\rho)$ (the superscript $i$ is used to denote the reference basis) is said to be a valid measure of quantum coherence of the state $\rho$ if it satisfies following conditions: (1) $C^{(i)}(\rho)=0$ iff $\rho \in \mathcal{I}$. (2) $C^{(i)}(\rho)$ is non increasing under the incoherent operations, i.e., for any incoherent channel denoted by $\Lambda_{I}$, we have $C^{(i)}\left(\Lambda_{I}[\rho]\right) \leq C^{(i)}(\rho)$. (3) $C^{(i)}(\rho)$ decreases on an average under the selective incoherent operations, i.e., $\sum_{k} p_{k} C^{(i)}\left(\rho_{k}\right) \leq C^{(i)}(\rho)$, where $\rho_{k}=M_{k} \rho M_{k}^{+} / p_{k}, p_{k}=\operatorname{Tr}\left[M_{k} \rho M_{k}^{+}\right]$and $M_{k}$ are the Kraus elements of an incoherent channel. (4) $C^{(i)}(\rho)$ is a convex function of quantum states, i.e., $C^{(i)}\left(\sum_{k} p_{k} \rho_{k}\right) \leq \sum_{k} p_{k} C^{(i)}\left(\rho_{k}\right)$. It may be noted that the conditions (3) and (4) together imply condition (2). The bona fide measures of coherence include the relative entropy of coherence, the $l_{1}$ norm of coherence [2], the coherence of formation and the distillable coherence [7]. In this work, we will work only with the relative entropy of coherence, which for a density matrix $\rho$ and a reference basis $\{|i\rangle\}$, is defined as

$$
C^{(i)}(\rho)=S\left(\rho_{d}^{(i)}\right)-S(\rho)
$$


where $S(\rho)=-\operatorname{Tr}[\rho \log \rho]$ is the von Neumann entropy. Here and in the rest of the paper, all the logarithms are taken with respect to base 2. The superscript $i$ in $C^{(i)}(\rho)$ indicates that coherence is calculated in reference basis $\{|i\rangle\}$. If $\rho$ is a pure density operator and $\rho=|\psi\rangle\langle\psi|$, then we have $C^{(i)}(\rho)=S\left(\rho_{d}^{(i)}\right)$, where $\rho_{d}^{(i)}$ is the diagonal part of $\rho=|\psi\rangle\langle\psi|$ in the basis $\{|i\rangle\}$.

\section{Uncertainty Like Relation for Quantum Coherence Expressed in Two Different Non-Commuting Bases}

Let us first consider the relation between the coherence of a single $d$-level quantum system in a state $\rho$ expressed in two different non commuting bases. Let the sets $P=\{|i\rangle\langle i|\}$ and $Q=\{|a\rangle\langle a|\}$ denote two non-commuting projective measurements on the given quantum system. The state of the system after the projective measurements by $P$ and $Q$, is given, respectively, by

$$
\begin{aligned}
& \rho_{P}=\sum_{i} p_{i}|i\rangle\langle i| \text { and } \\
& \rho_{Q}=\sum_{a} q_{a}|a\rangle\langle a|
\end{aligned}
$$

where $p_{i}=\langle i|\rho| i\rangle$ and $q_{a}=\langle a|\rho| a\rangle$. Now the entropic uncertainty relation [58,59] for these two measurements reads

$$
H(P)+H(Q) \geq-2 \log C+S(\rho)
$$

where $H(P)=-\sum_{i} p_{i} \log p_{i}, H(Q)=-\sum_{a} q_{a} \log q_{a}, S(\rho)=-\operatorname{Tr}[\rho \log \rho]$ and $C=\max _{i, a}|\langle i \mid a\rangle|$. Also, $H(P)=S\left(\rho_{d}^{(i)}\right)$ and $H(Q)=S\left(\rho_{d}^{(a)}\right)$, where $\rho_{d}^{(i)}$ and $\rho_{d}^{(a)}$ are the diagonal parts of the density matrix $\rho$ in bases $\{|i\rangle\}$ and $\{|a\rangle\}$, respectively. Now, Equation (4) can be rewritten as

$$
C^{(i)}(\rho)+C^{(a)}(\rho) \geq-2 \log C-S(\rho)
$$

where $C^{(i)}(\rho)=S\left(\rho_{d}^{(i)}\right)-S(\rho)$ and $C^{(a)}(\rho)=S\left(\rho_{d}^{(a)}\right)-S(\rho)$ are the coherences of the density matrix $\rho$ in the bases $\{|i\rangle\}$ and $\{|a\rangle\}$, respectively. The above relation can be termed as the uncertainty relation for the coherences of a density matrix measured in two different (non-commuting) bases. Since the coherence of a quantum system is a basis dependent notion, above relation gives us insight about the coherences of a same density matrix measured in two different bases.

Now let us consider a bipartite quantum system in a state $\rho_{A B}$. Also, let $\rho_{B}=\operatorname{Tr}_{A}\left[\rho_{A B}\right]=$ $\sum_{\mu} \lambda_{\mu}|\mu\rangle\langle\mu|$, where $\lambda_{\mu}$ are the eigenvalues and $|\mu\rangle$ are the eigenvectors of $\rho_{B}$. Consider two projective measurements on the state $\rho_{A B}$, given by $P_{A B}=\{|i\rangle\langle i|\otimes| \mu\rangle\langle\mu|\}$ and $Q_{A B}=\{|a\rangle\langle a|\otimes| \mu\rangle\langle\mu|\}$. Note that these projective measurements do not disturb the density operator $\rho_{B}$. The state of the bipartite system after the projective measurements by $P_{A B}$ and $Q_{A B}$, is given, respectively, by

$$
\begin{aligned}
& \rho_{P R}=\sum_{i, \mu} p_{i \mu}|i\rangle\langle i|\otimes| \mu\rangle\langle\mu| \text { and } \\
& \rho_{Q T}=\sum_{a, \mu} q_{a \mu}|a\rangle\langle a|\otimes| \mu\rangle\langle\mu|
\end{aligned}
$$

where $p_{i \mu}=\left\langle i, \mu\left|\rho_{A B}\right| i, \mu\right\rangle$ and $q_{a \mu}=\left\langle a, \mu\left|\rho_{A B}\right| a, \mu\right\rangle$. Noting that the projective measurements always increase entropy, we have $S\left(\rho_{P R}\right) \geq S\left(\rho_{P B}\right)$ and similarly, $S\left(\rho_{Q T}\right) \geq S\left(\rho_{Q B}\right)$. Here, $\rho_{P B}=\sum_{i}|i\rangle\left\langle i\left|\otimes \mathbb{I} \rho_{A B}\right| i\right\rangle\langle i| \otimes \mathbb{I}$ and similarly $\rho_{Q B}=\sum_{a}|a\rangle\left\langle a\left|\otimes \mathbb{I} \rho_{A B}\right| a\right\rangle\langle a| \otimes \mathbb{I}$. Also note that $S\left(\rho_{R}\right)=S\left(\rho_{T}\right)=S\left(\rho_{B}\right)$. Therefore, $H(P \mid R) \geq H(P \mid B)$ and $H(Q \mid T) \geq H(Q \mid B)$, where $H(X \mid Y)=S\left(\rho_{X Y}\right)-S\left(\rho_{Y}\right)$. This gives

$$
\begin{aligned}
H(P \mid R)+H(Q \mid T) & \geq H(P \mid B)+H(Q \mid B) \\
& \geq-2 \log C+S(A \mid B)
\end{aligned}
$$


where the last inequality follows from entropic uncertainty relation in the presence of memory, first given in the Ref. [59], and $C=\max _{i, a}|\langle i \mid a\rangle|$. Now $H(P \mid R)=S\left(\rho_{P R}\right)-S\left(\rho_{B}\right)=$ $C^{(i \mu)}\left(\rho_{A B}\right)+S\left(\rho_{A B}\right)-S\left(\rho_{B}\right)=C^{(i \mu)}\left(\rho_{A B}\right)+S(A \mid B)$. Similarly, $H(Q \mid T)=C^{(a \mu)}\left(\rho_{A B}\right)+S(A \mid B)$. Therefore, Equation (8) becomes

$$
C^{(i \mu)}\left(\rho_{A B}\right)+C^{(a \mu)}\left(\rho_{A B}\right) \geq-2 \log C-S(A \mid B)
$$

For pure bipartite entangled states $S(A \mid B)$ is negative, and hence the above uncertainty like relation for quantum coherence is tightened. This is in contrast to the memory assisted uncertainty relation, where entanglement reduces the uncertainty. Therefore, in the presence of entanglement, quantum coherence and entropic uncertainty relation seemingly behave in opposite ways. The entropic uncertainty relation in the presence of quantum memory has been improved in Ref. [62] and using it, we have

$$
\begin{aligned}
H(P \mid R)+H(Q \mid T) & \geq H(P \mid B)+H(Q \mid B) \\
& \geq-2 \log C+S(A \mid B)-\max \{0, \mathcal{D}-\mathcal{J}\}
\end{aligned}
$$

where $\mathcal{D}$ is quantum discord across $A B$ partition [63-65] and $\mathcal{J}$ is the classical correlation [63-65]. Therefore the Equation (9) is improved to

$$
C^{(i \mu)}\left(\rho_{A B}\right)+C^{(a \mu)}\left(\rho_{A B}\right) \geq-2 \log C-S(A \mid B)-\max \{0, \mathcal{D}-\mathcal{J}\}
$$

If $\mathcal{D}<\mathcal{J}$, the bound in Equation (10) becomes even more tighter.

For a tripartite state $\rho_{A B D}$, one may ask how does the entanglement across $A B$ and $A D$ affects the uncertainty like relation for quantum coherence. Similar to Equation (9), one can have a uncertainty like relation for quantum coherence for the density operator $\rho_{A D}$, which is given by

$$
C^{(i v)}\left(\rho_{A D}\right)+C^{(a v)}\left(\rho_{A D}\right) \geq-2 \log C-S(A \mid D)
$$

It turns out that entanglement cannot tighten uncertainty like relation for quantum coherence across $A B$ and $A D$ at the same time. This is because, we have $S(A \mid B)+S(A \mid D) \geq 0$ for any tripartite quantum state, which follows from the strong subadditivity of the von Neumann entropy [66,67]. This shows that whenever we have $S(A \mid B)<0$, we have $S(A \mid D)>0$. This means that if one tightens the uncertainty like relation for quantum coherence across $A B$ partition, the uncertainty like relation for quantum coherence across $A D$ partition cannot be improved despite the presence of entanglement in the state $\rho_{A B D}$.

\section{Uncertainty Like Relation for Quantum Coherence Expressed in Many Different Non-Commuting Bases}

Let us first consider a single $d$-level quantum system in a state $\rho$. Let the sets $P_{1}=\left\{\left|i_{1}\right\rangle\left\langle i_{1}\right|\right\}$ and $P_{2}=\left\{\left|i_{2}\right\rangle\left\langle i_{2}\right|\right\}, \ldots, P_{n}=\left\{\left|i_{n}\right\rangle\left\langle i_{n}\right|\right\}$ denote $n$ non-commuting projective measurements on the given quantum system. The state of the system after the projective measurements by $P_{1}, \ldots, P_{n}$ is given, respectively, by $\rho_{P_{1}}=\sum_{i_{1}} p_{i_{1}}\left|i_{1}\right\rangle\left\langle i_{1}\left|, \ldots, \rho_{P_{n}}=\sum_{i_{n}} p_{i_{n}}\right| i_{n}\right\rangle\left\langle i_{n}\right|$, where $p_{i_{1}}=\left\langle i_{1}|\rho| i_{1}\right\rangle, \ldots, p_{i_{n}}=\left\langle i_{n}|\rho| i_{n}\right\rangle$. Now the entropic uncertainty relation [68] for multiple measurements read

$$
\sum_{i=1}^{n} H\left(P_{i}\right) \geq-\log b+(n-1) S(\rho)
$$


where $H\left(P_{k}\right)=-\sum_{i_{k}} p_{i_{k}} \log p_{i_{k^{\prime}}}(k=1, \ldots, n), S(\rho)=-\operatorname{Tr}[\rho \log \rho]$ and

$$
b=\max _{i_{n}}\left\{\sum_{i_{2}, i_{n-1}} \max _{i_{1}}\left[c\left(i_{1}, i_{2}\right)\right] \prod_{k=2}^{n-1} c\left(i_{k}, i_{k+1}\right)\right\}
$$

where $c\left(i_{k}, j_{l}\right)=\left|\left\langle i_{k} \mid j_{l}\right\rangle\right|^{2}$. Also, $H\left(P_{k}\right)=S\left(\rho_{d}^{\left(i_{k}\right)}\right)$ with $k=1, \ldots, n$, where $\rho_{d}^{\left(i_{k}\right)}$ are the diagonal parts of the density matrix $\rho$ in bases $\left\{\left|i_{k}\right\rangle\right\}$. Now, Equation (12) can be rewritten as

$$
\begin{aligned}
& \sum_{k=1}^{n} S\left(\rho_{d}^{\left(i_{k}\right)}\right) \geq-\log b+(n-1) S(\rho), \text { or } \\
& \sum_{k=1}^{n} C^{\left(i_{k}\right)}(\rho) \geq-\log b-S(\rho)
\end{aligned}
$$

where $C^{\left(i_{k}\right)}(\rho)=S\left(\rho_{d}^{\left(i_{k}\right)}\right)-S(\rho)$ is the coherences of the density matrix $\rho$ in the $\left\{\left|i_{k}\right\rangle\right\}$ basis.

Now let us consider a bipartite quantum system in a state $\rho_{A B}$, where $\rho_{B}=\operatorname{Tr}_{A}\left[\rho_{A B}\right]=$ $\sum_{\mu} \lambda_{\mu}|\mu\rangle\langle\mu|$, and $n$ projective measurements on the state $\rho_{A B}$, given by $P_{A B}^{i}=\left\{\left|i_{k}\right\rangle\left\langle i_{k}|\otimes| \mu\right\rangle\langle\mu|\right\}$ with $k=1, \ldots, n$. The state of the bipartite system after the projective measurements by $P_{A B}^{k}$ is given by

$$
\rho_{P_{k} R_{k}}=\sum_{i_{k}, \mu} p_{i_{k} \mu}\left|i_{k}\right\rangle\left\langle i_{k}|\otimes| \mu\right\rangle\langle\mu|
$$

where $p_{i_{k} \mu}=\left\langle i_{k}, \mu\left|\rho_{A B}\right| i_{k}, \mu\right\rangle$. Noting that the projective measurements always increase entropy, we have $S\left(\rho_{P_{k} R_{k}}\right) \geq S\left(\rho_{P_{k} B}\right)$, with $\rho_{P_{k} B}=\sum_{i_{k}}\left|i_{k}\right\rangle\left\langle i_{k}\left|\otimes \mathbb{I} \rho_{A B}\right| i_{k}\right\rangle\left\langle i_{k}\right| \otimes \mathbb{I}$. Also note that $S\left(\rho_{R_{k}}\right)=S\left(\rho_{B}\right)$. Therefore, $H\left(P_{k} \mid R_{k}\right) \geq H\left(P_{k} \mid B\right)$. Now,

$$
\begin{aligned}
\sum_{k=1}^{n} H\left(P_{k} \mid R_{k}\right) & \geq \sum_{k=1}^{n} H\left(P_{k} \mid B\right) \\
& \geq-\log b+(n-1) S(A \mid B)
\end{aligned}
$$

where the last inequality follows from the many measurement generalization of entropic uncertainty relation in presence of memory [59] and is obtained in Ref. [68], and $b$ is given by Equation (13). Now $H\left(P_{k} \mid R_{k}\right)=S\left(\rho_{P_{k} R_{k}}\right)-S\left(\rho_{B}\right)=C^{\left(i_{k} \mu\right)}\left(\rho_{A B}\right)+S\left(\rho_{A B}\right)-S\left(\rho_{B}\right)=C^{\left(i_{k} \mu\right)}\left(\rho_{A B}\right)+S(A \mid B)$. Therefore, Equation (16) becomes

$$
\sum_{k=1}^{n} C^{\left(i_{k} \mu\right)}\left(\rho_{A B}\right) \geq-\log b-S(A \mid B)
$$

For bipartite pure entangled states, the above inequality is tightened.

\section{Complementarity Like Relation for Quantum Coherence for a Bipartite State}

For a bipartite quantum system, there exists an optimal decomposition of every density matrix $\rho_{A B}$ of the bipartite system, called as the Lewenstein-Sanpera (LS) decomposition, in terms of a separable state and an entangled state [61,69-72], i.e.,

$$
\rho_{A B}=\lambda \rho_{s}+(1-\lambda) \rho_{e} ; \lambda \in[0,1]
$$

where $\rho_{s}$ is a separable state on the separable-entangled boundary and $\rho_{e}$ is an entangled state. The parameter $\lambda$ is taken to be optimal in the sense that any other decomposition of the form $\lambda^{\prime} \rho_{s}^{\prime}+\left(1-\lambda^{\prime}\right) \rho_{e}^{\prime}$ with $\lambda^{\prime} \in[0,1]$ and $\rho_{s} \neq \rho_{s}^{\prime}$ necessarily implies $\lambda^{\prime}<\lambda$. For optimal $\lambda, \rho_{s}$ is called the optimal separable approximation (OSA) of the state $\rho_{A B}$. For a bipartite qubit system the optimal LS decomposition becomes unique with the entangled part of the decomposition being a pure 
entangled projector [61]. Using the concavity of the von Neumann entropy, Equation (18) implies $S\left(\rho_{A B}\right) \geq \lambda S\left(\rho_{S}\right)$. Let us denote the quantum coherence of the bipartite state $\rho_{A B}$ in two different bases $\{|i j\rangle\}$ and $\{|a b\rangle\}$ as $C^{i j}\left(\rho_{A B}\right)$ and $C^{a b}\left(\rho_{A B}\right)$, respectively, then

$$
\begin{aligned}
C^{i j}\left(\rho_{A B}\right)+C^{a b}\left(\rho_{A B}\right) & =S\left(\rho_{d}^{i j}\right)+S\left(\rho_{d}^{a b}\right)-2 S\left(\rho_{A B}\right) \\
& \leq 2 \log d_{A} d_{B}-2 K\left(\rho_{A B}\right)
\end{aligned}
$$

where $K\left(\rho_{A B}\right)=\lambda S\left(\rho_{s}\right)+(1-\lambda) S\left(\rho_{e}\right)$ and $d_{A(B)}$ being the dimension of the system $A(B)$. This tightens the trivial upper bound on the sum of coherences, namely $2 \log d_{A} d_{B}$. For bipartite qubit systems, since the entangled part in the optimal separable approximation becomes pure, $K\left(\rho_{A B}\right)$ becomes $\lambda S\left(\rho_{S}\right)$ and we have

$$
C^{i j}\left(\rho_{A B}\right)+C^{a b}\left(\rho_{A B}\right) \leq 4-2 \lambda S\left(\rho_{s}\right)
$$

In the following, we provide some examples of optimal LS decomposition and calculate the bound on the sum of the coherences in two different bases. Consider a $2 \otimes 2$ Bell diagonal state

$$
\rho_{A B}=\sum_{i=1}^{4} d_{i}\left|B_{i}\right\rangle\left\langle B_{i}\right|
$$

where $\left|B_{1}\right\rangle=(|00\rangle+|11\rangle) / \sqrt{2},\left|B_{2}\right\rangle=(|00\rangle-|11\rangle) / \sqrt{2},\left|B_{3}\right\rangle=(|01\rangle+|10\rangle) / \sqrt{2},\left|B_{4}\right\rangle=(|01\rangle-|10\rangle) / \sqrt{2}$ and $\left\{d_{i}\right\}$ is a probability vector. The optimal LS decomposition, $\rho_{A B}=\lambda \rho_{s}+(1-\lambda) \rho_{e}$ of the above Bell diagonal state is obtained for $\rho_{e}=\left|B_{1}\right\rangle\left\langle B_{1}\right|$ and $\rho_{s}=\sum_{i=1}^{4} d_{i}^{\prime}\left|B_{i}\right\rangle\left\langle B_{i}\right|$ where $d_{1}^{\prime}=1 / 2$, $d_{j}^{\prime}=d_{j} / \lambda(j=2,3,4)$ and $\lambda=1-\mu$ [70]. Here, $\mu$ is the concurrence [73,74] of the initial state $\rho_{A B}$. The quantity $K\left(\rho_{A B}\right)$, here, is given by $\frac{(1-\mu)}{2} \log 2+\left(1-d_{1}\right) \log (1-\mu)-\sum_{i=2}^{4} d_{i} \log d_{i}$. For a specific example, take $d_{1}=0.6, d_{2}=0.2, d_{3}=0.1=d_{4}$. In this case $\mu=0.2$ and $K\left(\rho_{A B}\right)=1.4$, where $\log$ is calculated using base 2 . Therefore, sum of the coherences for the Bell diagonal state, given by above parameters, satisfy

$$
C^{i j}\left(\rho_{A B}\right)+C^{a b}\left(\rho_{A B}\right) \leq 1.2
$$

This bound gives a value which is well below the trivial bound which is four. Another example that we consider is the case of bipartite qutrit state which contains bound entanglement [75] in it. The state is given by

$$
\rho=\frac{2}{7}\left|\psi_{+}\right\rangle\left\langle\psi_{+}\right|+\frac{\gamma}{7} P_{+}+\frac{5-\gamma}{7} P_{-} ; \quad 2 \leq \gamma \leq 5
$$

where $\left|\psi_{+}\right\rangle=(|00\rangle+|11\rangle+|22\rangle) / \sqrt{3}, \quad P_{+}=(|01\rangle\langle 01|+| 12\rangle\langle 12|+| 20\rangle\langle 20|) / 3$ and $P_{-}=(|10\rangle\langle 10|+| 21\rangle\langle 21|+| 02\rangle\langle 02|) / 3$. The optimal LS decomposition, $\rho=\lambda \rho_{s}+\left(1-\lambda \rho_{e}\right)$ of the state $\rho$ is obtained for $\rho_{e}=\frac{2}{7}\left|\psi_{+}\right\rangle\left\langle\psi_{+}\left|+\frac{5}{7} P_{+}, \rho_{s}=\frac{2}{7}\right| \psi_{+}\right\rangle\left\langle\psi_{+}\right|+\frac{3}{7} P_{+}+\frac{2}{7} P_{-}$and $\lambda=(5-\gamma) / 2$ [70]. The quantity $K\left(\rho_{A B}\right)$, here, is given by $0.8631+0.6935 \lambda$. Therefore, sum of the coherences for the state $\rho$, satisfy

$$
C^{i j}\left(\rho_{A B}\right)+C^{a b}\left(\rho_{A B}\right) \leq 2 \log 9-2(0.8631+0.6935 \lambda)
$$

The bound, Equation (19), can be tightened as follows. Let us consider a bipartite state $\rho_{A B}$. Also, assume that the marginal density matrix $\rho_{B}$ for subsystem $B$ is diagonal in the basis $|\mu\rangle$, i.e., 
$\rho_{B}=\sum_{\mu} d_{\mu}|\mu\rangle\langle\mu|$. Now let us consider a projective measurement on the bipartite system in the basis $\{|i\rangle \otimes|\mu\rangle\}$. The state of the total system after the measurement, is given by

$$
\rho_{P R}=\sum_{i, \mu}\left\langle i, \mu\left|\rho_{A B}\right| i, \mu\right\rangle|i, \mu\rangle\langle i, \mu|
$$

The marginal density matrix $\rho_{R}$ of the subsystem $B$ after the measurement is same as $\rho_{B}$. Now, $H(P \mid R)=H(P R)-H(R)=S\left(\rho_{P R}\right)-S\left(\rho_{B}\right)=C^{(i \mu)}\left(\rho_{A B}\right)+S(A \mid B)$. Therefore,

$$
\begin{aligned}
C^{(i \mu)}\left(\rho_{A B}\right) & =H(P \mid R)-S(A \mid B)=H(P)-I(P: R)-S(A \mid B) \\
& \leq H(P)-S(A \mid B) \\
& \leq \log d_{A}-S(A \mid B)
\end{aligned}
$$

where $d_{A}$ is the dimension of the subsystem $A$. Now, if we consider the projective measurement of the total system in the basis $\{|a, \mu\rangle\}$, we get

$$
C^{(a \mu)}\left(\rho_{A B}\right) \leq \log d_{A}-S(A \mid B)
$$

Therefore,

$$
C^{(i \mu)}\left(\rho_{A B}\right)+C^{(a \mu)}\left(\rho_{A B}\right) \leq 2 \log d_{A}-2 S(A \mid B)
$$

Again we consider the example of the Bell diagonal state $\rho_{A B}=\sum_{i=1}^{4} d_{i}\left|B_{i}\right\rangle\left\langle B_{i}\right|$, where $\left|B_{1}\right\rangle=(|00\rangle+|11\rangle) / \sqrt{2},\left|B_{2}\right\rangle=(|01\rangle+|10\rangle) / \sqrt{2},\left|B_{3}\right\rangle=(|01\rangle-|10\rangle) / \sqrt{2}$, and $\left|B_{4}\right\rangle=(|00\rangle+|11\rangle) / \sqrt{2}$. For $d_{1}>1 / 2$, the optimal LS decomposition is given by: $\lambda=2\left(1-d_{1}\right)$, $\rho_{e}=\left|B_{1}\right\rangle\left\langle B_{1}\right|$, and

$$
\rho_{s}=\sum_{i=1}^{4} d_{i}^{\prime}\left|B_{i}\right\rangle\left\langle B_{i}\right|
$$

where $d_{1}^{\prime}=1 / 2, d_{j}^{\prime}=d_{j} / \lambda(j=2,3,4)$. Note that the previous bound in Equation (19) is given by

$$
\begin{aligned}
& 2 \log d_{A} d_{B}-\lambda S\left(\rho_{s}\right) \\
& =4-\left(1-d_{1}\right)\left(2+\log \left[1-d_{1}\right]\right)+\sum_{i=2}^{4} d_{i} \log d_{i}
\end{aligned}
$$

However, the bound in Equation (28) is given by

$$
\begin{aligned}
2 \log d_{A}-2 S(A \mid B) & =2-2 S\left(\rho_{A B}\right)+2 S(B) \\
& =4+2 \sum_{i=1}^{4} d_{i} \log d_{i}
\end{aligned}
$$

For Bell diagonal states with $d_{1}>1 / 2$, the new bound (Equation (28)) is always smaller than the bound (Equation (19)) (see also Figure 1). 


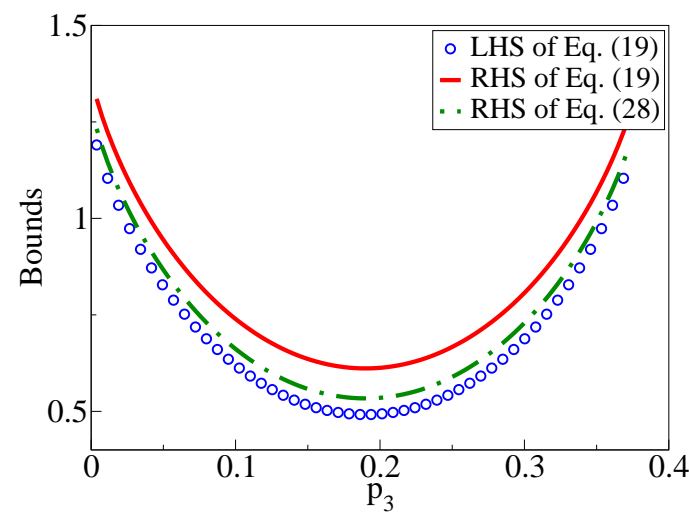

(a) $d_{1}=0.52, d_{2}=0.1$

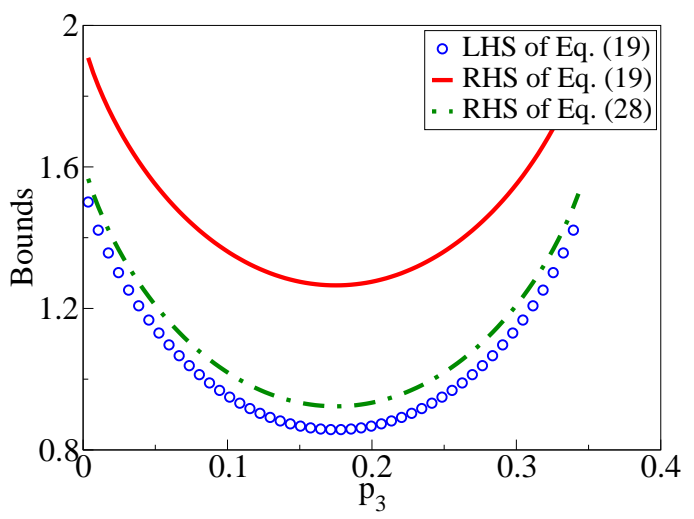

(b) $d_{1}=0.6, d_{2}=0.05$

Figure 1. The plot shows the left hand and right hand sides of the uncertainty like relations for quantum coherence, given by Equations (19) and (28), for Bell diagonal states. It clearly shows that the bound in Equation (28) is tighter than that in Equation (19). (a) $d_{1}=0.52, d_{2}=0.1 ;(\mathbf{b}) d_{1}=0.6$, $d_{2}=0.05$.

\section{State Dependent Upper Bound for Coherence}

One may ask that if diagonal parts of a density operator in two different reference bases are close, does this mean the closeness of coherences of the density operator in the given two reference bases? Let us consider a quantum system in a state $\rho$, with $\rho_{d}^{(i)}$ being the diagonal part of the density matrix in $\{|i\rangle\}$ basis. For, $\left\|\rho_{d}^{(i)}-\rho\right\|_{1}=2 \epsilon$, with $\|.\|_{1}$ being the trace norm, defined as $\|M\|_{1}=\operatorname{Tr} \sqrt{M^{+} M}$, the Fannes-Audenaert inequality [76,77] implies

$$
C^{(i)}(\rho)=\left|S\left(\rho_{d}^{(i)}\right)-S(\rho)\right| \leq \epsilon \log (d-1)+H(\epsilon)
$$

where $d$ is the dimension of the Hilbert space of the quantum system and $H(\epsilon)$ is the binary entropy. Let the diagonal part of the density matrix $\rho$ in the basis $\{|a\rangle\}$ be denoted by $\rho_{d}^{(a)}$. For $\left\|\rho_{d}^{(i)}-\rho_{d}^{(a)}\right\|_{1}=2 \eta$, again using the Fannes-Audenaert inequality $[66,76,77]$, we have

$$
\left|S\left(\rho_{d}^{(i)}\right)-S\left(\rho_{d}^{(a)}\right)\right| \leq \eta \log (d-1)+H(\eta)
$$

Note that $S\left(\rho_{d}^{(i)}\right)-S\left(\rho_{d}^{(a)}\right)=\left[S\left(\rho_{d}^{(i)}\right)-S(\rho)\right]-\left[S\left(\rho_{d}^{(a)}\right)-S(\rho)\right]=C^{(i)}(\rho)-C^{(a)}(\rho)$. Now, Equation (33) implies

$$
\left|C^{(i)}(\rho)-C^{(a)}(\rho)\right| \leq \eta \log (d-1)+H(\eta)
$$

When $\eta=\left\|\rho_{d}^{(i)}-\rho_{d}^{(a)}\right\|_{1} / 2$ is small, i.e., the diagonal parts of a quantum state in two different bases are close to each other with respect to the trace distance, the relative entropies of coherence of the state in these two bases are also close to each other.

\section{Summary}

In quantum theory, uncertainty relation is a fundamental consequence of superposition principle and incompatible nature of observables. As quantum coherence is a basis dependent notion, it is pertinent to ask if coherence respects some kind of uncertainty relation for two or more incompatible bases. In this paper, we have explored the interplay of the relative entropy of coherence of a quantum system in a given state in two or more incompatible bases. We have proved trade-off relations for the relative entropy of coherence in two or more non-commuting bases for single and bipartite quantum systems. This shows that the relative entropies of coherence of a quantum system in two or more 
incompatible bases are not independent of one another. If, in one basis, the density matrix shows a larger value of the relative entropy of coherence, in another basis it may not show the same value. In the case of bipartite states, the presence of entanglement tightens the trade-off relation for the relative entropy of coherence. However, because of strong subadditivity of conditional entropy, one cannot have tightened trade-off relations for quantum coherence measure across two different parties. Also, we have proved complementarity like relations for the relative entropy of coherence in two different bases. Moreover, we have provided an upper bound on the absolute value of the differences of the relative entropy of coherence obtained in two different bases.

Acknowledgments: M.N.B. acknowledges financial support from the John Templeton Foundation, the Spanish MINECO (FIS2013-46768, FIS2008-01236, and FIS2013-40627-P) with the support of FEDER funds, "Severo Ochoa" Programme (SEV-2015-0522), the Generalitat de Catalunya (2014-SGR-874 and 2014-SGR-966), and Fundació Privada Cellex. U.S. acknowledges the research fellowship of Department of Atomic Energy, Government of India.

Author Contributions: The main ideas were developed by all authors. All authors formulated and proved the main technical claims. All authors wrote the manuscript

Conflicts of Interest: The authors declare no conflict of interest.

\section{References}

1. Aberg, J. Quantifying Superposition. 2006, arXiv:quant-ph/0612146. arXiv.org e-Print archive. Available online: http:/ /arxiv.org/abs/quant-ph/0612146 (accessed 17 on December 2006).

2. Baumgratz, T.; Cramer, M.; Plenio, M.B. Quantifying Coherence. Phys. Rev. Lett. 2014, 113, 140401.

3. Girolami, D. Observable Measure of Quantum Coherence in Finite Dimensional Systems. Phys. Rev. Lett. 2014, 113, 170401.

4. Bromley, T.R.; Cianciaruso, M.; Adesso, G. Frozen Quantum Coherence. Phys. Rev. Lett. 2015, 114, 210401.

5. Streltsov, A.; Singh, U.; Dhar, H.S.; Bera, M.N.; Adesso, G. Measuring Quantum Coherence with Entanglement. Phys. Rev. Lett. 2015, 115, 020403.

6. Xi, Z.; Li, Y.; Fan, H. Quantum coherence and correlations in quantum system. Sci. Rep. 2015, 5, 10922.

7. Winter, A.; Yang, D. Operational Resource Theory of Coherence. Phys. Rev. Lett. 2016, 116, 120404.

8. Shao, L.H.; Xi, Z.; Fan, H.; Li, Y. Fidelity and trace-norm distances for quantifying coherence. Phys. Rev. A 2015, 91, 042120.

9. Pires, D.P.; Céleri, L.C.; Soares-Pinto, D.O. Geometric lower bound for a quantum coherence measure. Phys. Rev. A 2015, 91, 042330.

10. Du, S.; Bai, Z.; Guo, Y. Conditions for coherence transformations under incoherent operations. Phys. Rev. A 2015, 91, 052120.

11. Yao, Y.; Xiao, X.; Ge, L.; Sun, C.P. Quantum coherence in multipartite systems. Phys. Rev. A 2015, 92, 022112.

12. Killoran, N.; Steinhoff, F.E.S.; Plenio, M.B. Converting Nonclassicality into Entanglement. Phys. Rev. Lett. 2016, 116, 080402.

13. Zhang, L.; Pati, A.K.; Wu, J. Interference visibility, entanglement, and quantum correlation. Phys. Rev. A 2015, 92, 022316.

14. Singh, U.; Bera, M.N.; Dhar, H.S.; Pati, A.K. Maximally coherent mixed states: Complementarity between maximal coherence and mixedness. Phys. Rev. A 2015, 91, 052115.

15. Singh, U.; Bera, M.N.; Misra, A.; Pati, A.K. Erasing Quantum Coherence: An Operational Approach. 2015, arXiv:1506.08186. arXiv.org e-Print archive. Available online: http://arxiv.org/abs/1506.08186 (accessed on 26 June 2015).

16. Cheng, S.; Hall, M.J.W. Complementarity relations for quantum coherence. Phys. Rev. A 2015, 92, 042101.

17. Mondal, D.; Pramanik, T.; Pati, A.K. Steerability of Local Quantum Coherence. 2015, arXiv:1508.03770. arXiv.org e-Print archive. http:/ / arxiv.org/abs/1508.03770 (accessed 24 September 2015).

18. Mondal, D.; Datta, C.; Sazim, S. Quantum coherence sets the quantum speed limit for mixed states. Phys. Lett. A 2016, 380, $689-695$.

19. Kumar, A. Quantum Coherence and Its Distribution. 2015, arXiv:1508.00262. arXiv.org e-Print archive. Available online: http:/ /arxiv.org/abs/1508.00262 (accessed on 2 August 2015). 
20. Mani, A.; Karimipour, V. Cohering and decohering power of quantum channels. Phys. Rev. A 2015, 92, 032331.

21. Bu, K.; Zhang, L.; Wu, J. Cohering power of quantum operations. 2015, arXiv:1509.09109. arXiv.org e-Print archive. Available online: http:/ /arxiv.org/abs/1509.09109 (accessed on 30 September 2015).

22. Chitambar, E.; Streltsov, A.; Rana, S.; Bera, M.N.; Adesso, G.; Lewenstein, M. Assisted Distillation of Quantum Coherence. Phys. Rev. Lett. 2016, 116, 070402.

23. Streltsov, A.; Rana, S.; Bera, M.N.; Lewenstein, M. Hierarchies of incoherent quantum operations. 2015, arXiv:1509.07456. arXiv.org e-Print archive. Available online: http://arxiv.org/abs/1509.07456 (accessed on 24 September 2015).

24. Chitambar, E.; Hsieh, M.-H. Relating the Resource Theories of Entanglement and Quantum Coherence. Phys. Rev. Lett. 2016, 117, 020402.

25. Streltsov, A. Genuine Quantum Coherence. 2015, arXiv:1511.08346. arXiv.org e-Print archive. Available online: http:/ / arxiv.org/abs/1511.08346 (accessed on 26 November 2015).

26. Bera, M.N.; Qureshi, T.; Siddiqui, M.A.; Pati, A.K. Duality of quantum coherence and path distinguishability. Phys. Rev. A 2015, 92, 012118.

27. Bagan, E.; Bergou, J.A.; Cottrell, S.S.; Hillery, M. Relations between Coherence and Path Information. Phys. Rev. Lett. 2016, 116, 160406.

28. Streltsov, A.; Chitambar, E.; Rana, S.; Bera, M.N.; Winter, A.; Lewenstein, M. Entanglement and Coherence in Quantum State Merging. Phys. Rev. Lett. 2016, 116, 240405.

29. Hillery, M. Coherence as a resource in decision problems: The Deutsch-Jozsa algorithm and a variation. Phys. Rev. A 2016, 93, 012111.

30. Rana, S.; Parashar, P.; Lewenstein, M. Trace-distance measure of coherence. Phys. Rev. A 2016, 93, 012110.

31. Rastegin, A.E. Quantum-coherence quantifiers based on the Tsallis relative $\alpha$ entropies. Phys. Rev. A 2016, 93, 032136.

32. Rodríguez-Rosario, C.A.; Frauenheim, T.; Aspuru-Guzik, A. Thermodynamics of quantum coherence. 2013, arXiv:1308.1245. arXiv.org e-Print archive. http://arxiv.org/abs/1308.1245 (accessed 6 August 2013).

33. Horodecki, M.; Oppenheim, J. Fundamental limitations for quantum and nanoscale thermodynamics. Nat. Commun. 2013, 4, 2059.

34. Skrzypczyk, P.; Short, A.J.; Popescu, S. Work extraction and thermodynamics for individual quantum systems. Nat. Commun. 2014, 5, 4185.

35. Narasimhachar, V.; Gour, G. Low-temperature thermodynamics with quantum coherence. Nat. Commun. 2015, 6, 7689.

36. Brandão, F.; Horodecki, M.; Ng, N.; Oppenheim, J.; Wehner, S. The second laws of quantum thermodynamics. Proc. Natl. Acad. Sci. USA 2015, 112, 3275-3279.

37. Lostaglio, M.; Jennings, D.; Rudolph, T. Description of quantum coherence in thermodynamic processes requires constraints beyond free energy. Nat. Commun. 2015, 6, 6383.

38. Lostaglio, M.; Korzekwa, K.; Jennings, D.; Rudolph, T. Quantum Coherence, Time-Translation Symmetry, and Thermodynamics. Phys. Rev. X 2015, 5, 021001.

39. Ćwikliński, P.; Studziński, M.; Horodecki, M.; Oppenheim, J. Limitations on the Evolution of Quantum Coherences: Towards Fully Quantum Second Laws of Thermodynamics. Phys. Rev. Lett. 2015, 115, 210403.

40. Gardas, B.; Deffner, S. Thermodynamic universality of quantum Carnot engines. Phys. Rev. E 2015, $92,042126$.

41. Misra, A.; Singh, U.; Bera, M.N.; Rajagopal, A.K. Quantum Rényi relative entropies affirm universality of thermodynamics. Phys. Rev. E 2015, 92, 042161.

42. Goold, J.; Huber, M.; Riera, A.; del Rio, L.; Skrzypczyk, P. The role of quantum information in thermodynamics-A topical review. 2015, arXiv:1505.07835. arXiv.org e-Print archive. Available online: http:/ / arxiv.org/abs/1505.07835 (accessed on 28 May 2015).

43. Abbott, D.; Davies, P.C.W.; Pati, A.K. Quantum Aspects of Life; Imperial College Press: London, UK, 2008.

44. Plenio, M.B.; Huelga, S.F. Dephasing-assisted transport: quantum networks and biomolecules. New J. Phys. 2008, 10, 113019.

45. Levi, F.; Mintert, F. A quantitative theory of coherent delocalization. New J. Phys. 2014, 16, 033007. 
46. Rebentrost, P.; Mohseni, M.; Aspuru-Guzik, A. Role of quantum coherence and environmental fluctuations in chromophoric energy transport. J. Phys. Chem. B 2009, 113, 9942.

47. Lloyd, S. Quantum coherence in biological systems. J. Phys. Conf. Ser. 2011, 302, 012037.

48. Li, C.M.; Lambert, N.; Chen, Y.N.; Chen, G.Y.; Nori, F. Witnessing Quantum Coherence: From solid-state to biological systems. Sci. Rep. 2012, 2, 885.

49. Huelga, S.; Plenio, M. Vibrations, quanta and biology. Contemp. Phys. 2013, 54, 181-207.

50. Heisenberg, W. Über den anschaulichen Inhalt der quantentheoretischen Kinematik und Mechanik. Z. Phys. 1927, 43, 172-198.

51. Kennard, E.H. Zur Quantenmechanik einfacher Bewegungstypen. Z. Phys. 1927, 44, 326-352.

52. Robertson, H.P. The Uncertainty Principle. Phys. Rev. 1929, 34, 163-164.

53. Schrödinger, E. Zum Heisenbergschen Unschärfeprinzip. Ber. Kgl. Akad. Wiss. Berlin 1930, $24,296$.

54. Maccone, L.; Pati, A.K. Stronger Uncertainty Relations for All Incompatible Observables. Phys. Rev. Lett. 2014, 113, 260401.

55. Busch, P.; Heinonen, T.; Lahti, P. Heisenberg's uncertainty principle. Phys. Rep. 2007, 452, 155-176.

56. Białynicki-Birula, I.; Mycielski, J. Uncertainty relations for information entropy in wave mechanics. Commun. Math. Phys. 1975, 44, 129-132.

57. Deutsch, D. Uncertainty in Quantum Measurements. Phys. Rev. Lett. 1983, 50, 631-633.

58. Maassen, H.; Uffink, J.B.M. Generalized entropic uncertainty relations. Phys. Rev. Lett. 1988, 60, 1103-1106.

59. Berta, M.; Christandl, M.; Colbeck, R.; Renes, J.M.; Renner, R. The uncertainty principle in the presence of quantum memory. Nat. Phys. 2010, 6, 659-662.

60. Coles, P.J.; Berta, M.; Tomamichel, M.; Wehner, S. Entropic Uncertainty Relations and their Applications. 2015, arXiv:1511.04857. arXiv.org e-Print archive. Available online: http://arxiv.org/abs/1511.04857 (accessed on 16 November 2015).

61. Lewenstein, M.; Sanpera, A. Separability and Entanglement of Composite Quantum Systems. Phys. Rev. Lett. 1998, 80, 2261-2264.

62. Pati, A.K.; Wilde, M.M.; Devi, A.R.U.; Rajagopal, A.K.; Sudha. Quantum discord and classical correlation can tighten the uncertainty principle in the presence of quantum memory. Phys. Rev. A 2012, 86, 042105

63. Henderson, L.; Vedral, V. Classical, quantum and total correlations. J. Phys. A Math. Gen. 2001, 34, 6899.

64. Ollivier, H.; Zurek, W.H. Quantum Discord: A Measure of the Quantumness of Correlations. Phys. Rev. Lett. 2001, 88, 017901.

65. Modi, K.; Brodutch, A.; Cable, H.; Paterek, T.; Vedral, V. The classical-quantum boundary for correlations: Discord and related measures. Rev. Mod. Phys. 2012, 84, 1655-1707.

66. Nielsen, M.A.; Chuang, I.L. Quantum Computation and Quantum Information; Cambridge University Press: Cambridge, UK, 2010.

67. Hu, M.L.; Fan, H. Competition between quantum correlations in the quantum-memory-assisted entropic uncertainty relation. Phys. Rev. A 2013, 87, 022314.

68. Liu, S.; Mu, L.Z.; Fan, H. Entropic uncertainty relations for multiple measurements. Phys. Rev. A 2015, 91, 042133.

69. Karnas, S.; Lewenstein, M. Separable approximations of density matrices of composite quantum systems. J. Phys. A Math. Gen. 2001, 34, 6919.

70. Akhtarshenas, S.J.; Jafarizadeh, M.A. Optimal Lewenstein-Sanpera decomposition for some bipartite systems. J. Phys. A Math. Gen. 2004, 37, 2965.

71. Thiang, G.C.; Raynal, P.; Englert, B.G. Optimal Lewenstein-Sanpera decomposition of two-qubit states using semidefinite programming. Phys. Rev. A 2009, 80, 052313.

72. Thiang, G.C. Degree of separability of bipartite quantum states. Phys. Rev. A 2010, 82, 012332.

73. Hill, S.; Wootters, W.K. Entanglement of a Pair of Quantum Bits. Phys. Rev. Lett. 1997, 78, 5022-5025.

74. Wootters, W.K. Entanglement of Formation of an Arbitrary State of Two Qubits. Phys. Rev. Lett. 1998, 80, 2245-2248.

75. Horodecki, M.; Horodecki, P. Reduction criterion of separability and limits for a class of distillation protocols. Phys. Rev. A 1999, 59, 4206-4216. 
76. Fannes, M. A continuity property of the entropy density for spin lattice systems. Commun. Math. Phys. 1973, 31, 291-294.

77. Audenaert, K.M.R. A sharp continuity estimate for the von Neumann entropy. J. Phys. A Math. Theor. 2007, 40,8127 .

(c)

(C) 2016 by the authors; licensee MDPI, Basel, Switzerland. This article is an open access article distributed under the terms and conditions of the Creative Commons Attribution (CC-BY) license (http://creativecommons.org/licenses/by/4.0/). 